\title{
A DoF Analysis of Compress-and-Forward in MIMO Gaussian Relay Channel with Correlated Noises
}

\author{
Seyed Arvin Ayoughi and Wei Yu \\ Department of Electrical and Computer Engineering \\ University of Toronto, Toronto, ON, M5S 3G4, Canada \\ Emails: sa.ayoughi@mail.utoronto.ca,weiyu@comm.utoronto.ca
}

\begin{abstract}
This paper studies the effectiveness of compress-andforward (CF) relaying scheme for a multiple-input multiple-output (MIMO) Gaussian relay channel with an out-of-band finite-capacity relay-to-destination link in which noises at the relay and destination are correlated due to common sources of interference. This scenario is motivated by the possibility of using device-to-device links for inter-cell interference mitigation in cellular networks. We characterize the necessary and sufficient conditions on the number of antennas under which the relay link rate can result in a near oneto-one improvement to the overall throughput in the high signalto-noise-ratio and interference-to-noise-ratio regime. We show that these conditions coincide with the necessary and sufficient condition under which full cooperation between relay and destination can improve the degrees of freedom of the relay channel. Simultaneous diagonalization by *congruence enables this characterization.
\end{abstract}

\section{INTRODUCTION}

This paper studies the effectiveness of compress-and-forward (CF) relaying strategy for interference mitigation in cellular networks. The scenario consists of a Gaussian multiple-input multiple-output (MIMO) relay channel with an out-of-band relay-to-destination link of finite capacity $C_{0}$, where the noises at the relay and destination are correlated due to common sources of interference. The idea is that cooperative relaying using device-to-device link between the mobile users can enable interference rejection for cell-edge users.

We measure the performance of CF in two different ways. First, we study the slope of $\mathrm{CF}$ achievable rate as a function of $C_{0}$ at $C_{0}=0$, and characterize the necessary and sufficient condition on the number of antennas in the network under which this slope approaches its maximum value of 1 in the high signal-to-noise ratio (SNR) and interference-to-noise ratio (INR) regime. Second, we study the possibility of improving degrees of freedom (DoF) due to relaying at $C_{0}=\infty$. Interestingly, the condition on the number of antennas under which the aforementioned slope approaches one is exactly the same condition under which relaying with $C_{0}=\infty$ improves DoF.

For the MIMO relay channel with independent noises, CF is studied in [1] [2]. This paper focuses on MIMO relaying in presence of noise correlation due to common interference sources, and follows our previous work [3] in using a simultaneous diagonalization by * congruence approach to find the optimal compression strategy at the relay. The optimization of quantization scheme in MIMO $\mathrm{CF}$ with correlated noises is connected to certain source coding problem in [4]. Although the solutions in [4] and [3] can be shown to be the same, the simultaneous diagonalization technique of [3] is simpler and provides more insight on the performance of MIMO CF for interference management. Specifically, this diagonalization enables the characterization of slope of $\mathrm{CF}$ rate as a function of the maximal generalized eigenvalue of two conditional covariance matrices, and reveals that relaying is most effective whenever there is a deterministic component in the underlying vector Gaussian relay channel. The determinism here refers to the condition that the observation of the relay is a deterministic function of the input of the channel and the observation at the destination. As shown in [5], CF achieves the cut-set bound in this case. This paper illustrates that this type of determinism happens for the MIMO relay channel with correlated interference in the high SNR and INR regime, and this is the fundamental reason that $\mathrm{CF}$ relaying can be effective in improving the overall throughput in an interference limited regime.

\section{System Model And Main Results}

The system model under study is depicted in Fig. 1. The number of antennas at the source, relay, and destination is denoted by $s, r$, and $d$, respectively. The relay and destination observe interference from common interference sources with total number of antennas equal to $t$. This channel is modeled mathematically as:

$$
\begin{gathered}
\mathbf{Y}_{r}=H_{s r} \mathbf{X}+H_{t r} \mathbf{X}_{t}+\mathbf{N}_{1}, \\
\mathbf{Y}_{d}=H_{s d} \mathbf{X}+H_{t d} \mathbf{X}_{t}+\mathbf{N}_{2},
\end{gathered}
$$

where $H_{s r} \in \mathbb{C}^{r \times s}$ and $H_{s d} \in \mathbb{C}^{d \times s}$ are source-relay and source-destination channel matrices respectively; $H_{t r} \in$ $\mathbb{C}^{r \times t}$ and $H_{t d} \in \mathbb{C}^{d \times t}$ are the interferers-to-relay and interferers-to-destination channel matrices respectively; $\mathbf{N}_{1} \sim$ $\mathcal{C N}\left(\mathbf{0}_{r \times 1}, \sigma^{2} \mathbf{I}_{r}\right)$ and $\mathbf{N}_{2} \sim \mathcal{C N}\left(\mathbf{0}_{d \times 1}, \sigma^{2} \mathbf{I}_{d}\right)$ are additive and independent background noises at the relay and the destination respectively. It is assumed that all channel matrices are full rank.

The transmit vector from the source is $\mathbf{X} \in \mathbb{C}^{s \times 1}$ and is assumed to have a fixed Gaussian distribution $\mathbf{X} \sim$ $\mathcal{C N}\left(\mathbf{0}_{s \times 1}, S_{\mathbf{X}}\right)$. The interference signal is denoted by $\mathbf{X}_{t} \in$ $\mathbb{C}^{t \times 1}$ with a fixed Gaussian distribution $\mathbf{X}_{t} \sim \mathcal{C N}\left(\mathbf{0}_{t \times 1}, S_{\mathbf{X}_{t}}\right)$, which is independent of other variables and is treated as noise. The matrices $S_{\mathbf{X}}$ and $S_{\mathbf{X}_{t}}$ are assumed to be full rank.

When the input distribution is fixed, the achievable rate of $\mathrm{CF}$ relaying scheme [6, Theorem 6] is:

$$
\begin{aligned}
R_{C F}\left(C_{0}\right)= & \max _{p\left(\widehat{\mathbf{y}}_{r} \mid \mathbf{y}_{r}\right)} I\left(\mathbf{X} ; \widehat{\mathbf{Y}}_{r}, \mathbf{Y}_{d}\right) \\
& \text { s.t. } I\left(\mathbf{Y}_{r} ; \widehat{\mathbf{Y}}_{r} \mid \mathbf{Y}_{d}\right) \leq C_{0}
\end{aligned}
$$




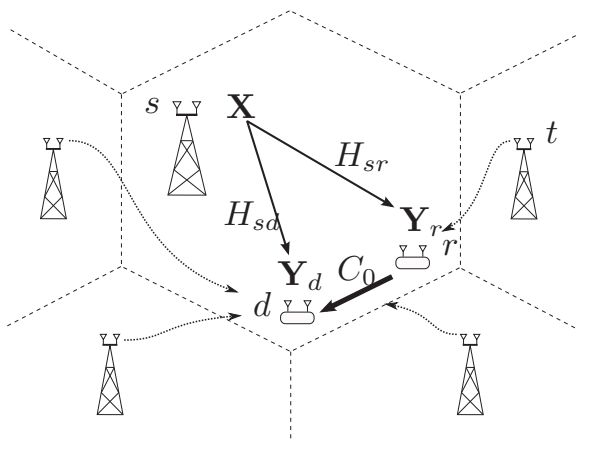

Fig. 1. A wireless cellular network with a base-station and two cell-edge users connected with a finite-capacity device-to-device cooperative relay link. The two users experience correlated noises due to common sources of interference.

In [4] it has been shown that when input distribution is Gaussian, Gaussian quantization is optimal. Thus, this paper restricts attention to Gaussian quantization, which is modeled as:

$$
\widehat{\mathbf{Y}}_{r}=\mathbf{Y}_{r}+\mathbf{Q}
$$

where $\mathbf{Q} \sim \mathcal{C N}\left(\mathbf{0}_{r \times 1}, S_{\mathbf{Q}}\right)$ is the quantization noise and is statistically independent of other variables in the network. In this case, the $\mathrm{CF}$ achievable rate can be characterized as:

$$
\begin{gathered}
R_{C F}\left(C_{0}\right)=\max _{S_{\mathbf{Q} \succ \mathbf{0}}} \log \frac{\mid S_{\mathbf{Y}_{r}\left|\mathbf{Y}_{d}+S_{\mathbf{Q}}\right|}}{\left|S_{\mathbf{Y}_{r} \mid \mathbf{Y}_{d}, \mathbf{X}}+S_{\mathbf{Q}}\right|}+I\left(\mathbf{X} ; \mathbf{Y}_{d}\right) \\
\text { s.t. } \log \frac{\left|S_{\mathbf{Y}_{r}\left|\mathbf{Y}_{d}+S_{\mathbf{Q}}\right|} \leq C_{0}\right|}{\left|S_{\mathbf{Q}}\right|}
\end{gathered}
$$

where the expressions of the conditional covariances $S_{\mathbf{Y}_{r} \mid \mathbf{Y}_{d}}$ and $S_{\mathbf{Y}_{r} \mid \mathbf{Y}_{d}, \mathbf{X}}$ can be found in [3], and $I\left(\mathbf{X} ; \mathbf{Y}_{d}\right)$ is a constant.

Cut-set bound provides an upper bound on the capacity of the relay channel [6, Theorem 4$]$, which is denoted by $\mathcal{C}$ :

$$
\mathcal{C}\left(C_{0}\right) \leq \min \left\{I\left(\mathbf{X} ; \mathbf{Y}_{r}, \mathbf{Y}_{d}\right), I\left(\mathbf{X} ; \mathbf{Y}_{d}\right)+C_{0}\right\}
$$

under a fixed distribution $p(\mathbf{x}) p\left(\mathbf{y}_{d}, \mathbf{y}_{r} \mid \mathbf{x}\right)$.

\section{A. The Main Result}

We consider two measures of performance for relaying: the possibility of improving DoF by relaying with $C_{0}=\infty$, and the slope of $R_{C F}\left(C_{0}\right)$ at $C_{0}=0$.

At $C_{0}=\infty$ the decoder at the destination has access to the relay's observations. This is equivalent to a genie that provides the destination with $r$ more antennas. The direct transmission capacity is $R_{C F}(0)=I\left(\mathbf{X} ; \mathbf{Y}_{d}\right)$; the genie-aided relaying capacity is $R_{C F}(\infty)=I\left(\mathbf{X} ; \mathbf{Y}_{r}, \mathbf{Y}_{d}\right)$. The DoF of the system with and without genie-aided relaying can be obtained by counting the number of antennas, i.e.,

$$
\operatorname{DoF}_{\mathrm{R}}=\lim _{\sigma^{2} \rightarrow 0} \frac{R_{C F}(\infty)}{-\log \left(\sigma^{2}\right)}=\min \left(s,(r+d-t)^{+}\right),
$$

and

$$
\operatorname{DoF}_{\mathrm{D}}=\lim _{\sigma^{2} \rightarrow 0} \frac{R_{C F}(0)}{-\log \left(\sigma^{2}\right)}=\min \left(s,(d-t)^{+}\right) .
$$

A simple analysis reveals that $\operatorname{DoF}_{R}>\operatorname{DoF}_{D}$ if and only if:

$$
d<s+t \text { and } r+d>t .
$$

To interpret the above conditions, we note that in order to improve DoF by genie-aided relaying, destination by itself must have less antennas than that deeded for canceling all of the interference while still having enough dimensions left for the intended signal. Thus, $d<s+t$ is required. Furthermore, to improve DoF with genie-aided relaying, the relay and the destination together must have enough antennas to cancel all of the interference. Thus, we need $r+d>t$. Otherwise genie-aided relaying cannot improve the spatial DoF.

At $C_{0}=0$, we study $\left.\frac{d R_{C F}}{d C_{0}}\right|_{C_{0}=0}$. Note that $R_{C F}\left(C_{0}\right)$ is concave in $C_{0}$. Therefore, its maximum slope occurs at $C_{0}=$ 0 . Since $R_{C F}(0)=I\left(\mathbf{X} ; \mathbf{Y}_{d}\right)$ and $R_{C F}\left(C_{0}\right)$ is bounded by $I\left(\mathbf{X} ; \mathbf{Y}_{d}\right)+C_{0}$ in (6), the slope cannot exceed 1 .

The main result of this paper is the following theorem which relates (9) to the conditions under which $\left.\frac{d R_{C F}}{d C_{0}}\right|_{C_{0}=0}$ approaches 1. The proof is presented in the following section.

Theorem 1 We have

$$
\left.\lim _{\sigma^{2} \rightarrow 0} \frac{d R_{C F}}{d C_{0}}\right|_{C_{0}=0}=1
$$

if and only if $\mathrm{DoF}_{\mathrm{R}}>\mathrm{DoF}_{\mathrm{D}}$, i.e., $d<s+t$ and $r+d>t$.

\section{Optimization of $S_{\mathbf{Q}}$}

To characterize the slope in Theorem 1, we need to solve the optimization problem (5). As shown in [3], the optimal $S_{\mathbf{Q}}$ can be found in closed form. The key tool is the simultaneous diagonalization of two conditional covariance matrices $S_{\mathbf{Y}_{r} \mid \mathbf{Y}_{d}}$ and $S_{\mathbf{Y}_{r} \mid \mathbf{Y}_{d}, \mathbf{X} \text {. According to [3, Lemma 2], we can find a full }}$ rank matrix $C \in \mathbb{C}^{r \times r}$ such that $C^{\dagger} S_{\mathbf{Y}_{r} \mid \mathbf{Y}_{d}, \mathbf{X}} C=\mathbf{I}_{r}$ and $C^{\dagger} S_{\mathbf{Y}_{r} \mid \mathbf{Y}_{d}} C=\Lambda$, where $\Lambda$ is a diagonal matrix with diagonal elements $\lambda_{i} \geq 1$ for $i=1, \ldots, r$, and $\lambda_{i}$ is the $i^{\text {th }}$ largest eigenvalue of the matrix $S_{\mathbf{Y}_{r} \mid \mathbf{Y}_{d}} S_{\mathbf{Y}_{r} \mid \mathbf{Y}_{d}, \mathbf{X}}^{-1}$.

After simultaneous diagonalization of $S_{\mathbf{Y}_{r} \mid \mathbf{Y}_{d}, \mathbf{X}}$ and $S_{\mathbf{Y}_{r} \mid \mathbf{Y}_{d}}$ the optimization of $S_{\mathbf{Q}}$ in (5) can be written as:

$$
\begin{aligned}
& \max _{\Sigma_{\mathbf{Q}} \succ \mathbf{0}} \log \frac{\left|\Lambda+\Sigma_{\mathbf{Q}}\right|}{\left|\mathbf{I}_{r}+\Sigma_{\mathbf{Q}}\right|} \\
& \text { s.t. } \log \frac{\left|\Lambda+\Sigma_{\mathbf{Q}}\right|}{\left|\Sigma_{\mathbf{Q}}\right|} \leq C_{0},
\end{aligned}
$$

where $\Sigma_{\mathbf{Q}}=C^{\dagger} S_{\mathbf{Q}} C$. Using [2, Lemma 5] it can be shown that $\Sigma_{\mathrm{Q}}$ can be chosen to be diagonal without loss of optimality. Denote the diagonal elements of $\Sigma_{\mathbf{Q}}$ by $\Sigma_{\mathbf{Q}}^{i i}$. We can now use a change of variable, introduced in [1]:

$$
c_{i}=\log \left(1+\frac{\lambda_{i}}{\Sigma_{\mathbf{Q}}^{i i}}\right), i=1, \ldots, r
$$

to reformulate (11) as:

$$
\begin{array}{ll}
\max _{c_{i} \geq 0} & \sum_{i=1}^{r}\left(c_{i}-\log \left(\frac{2^{c_{i}}}{\lambda_{i}}+1-\frac{1}{\lambda_{i}}\right)\right) \\
\text { s.t. } & \sum_{i=1}^{r} c_{i} \leq C_{0} .
\end{array}
$$

Here $c_{i}$ is the portion of the available capacity $C_{0}$ to be assigned for quantization of the $i^{t h}$ element of $C \mathbf{Y}_{r}$. For $\lambda_{i} \geq 1$, it 
can readily be checked that (13) is a convex problem, and a globally optimal solution can be found by solving its KarushKuhn-Tucker (KKT) system. The global optimum solution of (13) is:

$$
c_{i}^{*}=\left[\log \frac{\left(1-\mu^{*}\right)\left(\lambda_{i}-1\right)}{\mu^{*}}\right]^{+}
$$

where $\mu^{*}$ is selected such that $\sum_{i=1}^{r} c_{i}^{*}=C_{0}$. Then we have $\Sigma_{\mathbf{Q}}^{i i, *}=\frac{\lambda_{i}}{2^{c_{i}^{*}-1}}$, and the globally optimal quantization noise covariance is given by $S_{\mathbf{Q}}^{*}=C^{-\dagger} \Sigma_{\mathbf{Q}}^{*} C^{-1}$.

\section{Slope of $R_{C F}\left(C_{0}\right)$ AT $C_{0}=0$}

In this section, we use the optimal $S_{\mathrm{Q}}^{*}$ found above to prove Theorem 1, i.e., to characterize the necessary and sufficient conditions on the structure of correlation between relay's and destination's noises for CF to approach cut-set-bound asymptotically. The two lemmas below directly imply Theorem 1 .

Lemma 2 The slope of $R_{C F}\left(C_{0}\right)$ at $C_{0}=0$ is equal to $1-$ $\frac{1}{\lambda_{1}}$, where $\lambda_{1}$ is the largest generalized eigenvalue obtained by

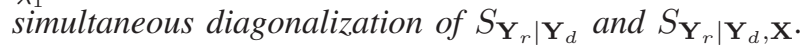

Proof: The optimal dual variable that satisfies the KKT conditions for a given $\bar{C}_{0}$ in (13), denoted by $\mu^{*}\left(\bar{C}_{0}\right)$, characterizes the slope of $R_{C F}\left(C_{0}\right)$ at $\bar{C}_{0}$. At $\bar{C}_{0}=0$ using the KKT solution (14), we have:

$$
\log \frac{\left(1-\mu^{*}(0)\right)\left(\lambda_{1}-1\right)}{\mu^{*}(0)}=\bar{C}_{0}=0 .
$$

Therefore,

$$
\left.\frac{d R_{C F}\left(C_{0}\right)}{d C_{0}}\right|_{C_{0}=0}=\mu^{*}(0)=1-\frac{1}{\lambda_{1}\left(S_{\mathbf{Y}_{r} \mid \mathbf{Y}_{d}} S_{\mathbf{Y}_{r} \mid \mathbf{Y}_{d}, \mathbf{X}}^{-1}\right)}
$$

Lemma 3 We have $\lim _{\sigma^{2} \rightarrow 0} \lambda_{1}\left(S_{\mathbf{Y}_{r} \mid \mathbf{Y}_{d}} S_{\mathbf{Y}_{r} \mid \mathbf{Y}_{d}, \mathbf{X}}^{-1}\right)=+\infty$ if and only if $d<s+t$ and $r+d>t$.

Proof: We take the limit of the maximal generalized eigenvalue through the squeeze theorem using the following bounds [7]:

$\max _{1 \leq i \leq r} \frac{\lambda_{i}\left(S_{\mathbf{Y}_{r} \mid \mathbf{Y}_{d}}\right)}{\lambda_{i}\left(S_{\mathbf{Y}_{r} \mid \mathbf{Y}_{d}, \mathbf{X}}\right)} \leq \lambda_{1}\left(S_{\mathbf{Y}_{r} \mid \mathbf{Y}_{d}} S_{\mathbf{Y}_{r} \mid \mathbf{Y}_{d}, \mathbf{X}}^{-1}\right) \leq \frac{\lambda_{1}\left(S_{\mathbf{Y}_{r} \mid \mathbf{Y}_{d}}\right)}{\lambda_{r}\left(S_{\mathbf{Y}_{r} \mid \mathbf{Y}_{d}, \mathbf{X}}\right)}$

Furthermore, define:

$$
\begin{gathered}
\overline{\mathbf{Y}}_{r} \triangleq H_{s r} \mathbf{X}+H_{t r} \mathbf{X}_{t} \\
\overline{\mathbf{Y}}_{d} \triangleq H_{s d} \mathbf{X}+H_{t d} \mathbf{X}_{t}
\end{gathered}
$$

as the received signal of the relay and destination without the background noise. Now, we use rank additivity formula for generalized Schur complement [8, Theorem 1] to relate the rank of $S_{\overline{\mathbf{Y}}_{r} \mid \overline{\mathbf{Y}}_{d}, \mathbf{X}}$ and $S_{\overline{\mathbf{Y}}_{r} \mid \overline{\mathbf{Y}}_{d}}$ to the number of antennas in the network.

Assuming $S_{\mathbf{X}}$ and $S_{\mathbf{X}_{t}}$ are full rank, it is easy to see that:

$$
\begin{aligned}
\operatorname{rank}\left(S_{\overline{\mathbf{Y}}_{r}, \overline{\mathbf{Y}}_{d}}\right) & =\min (r+d, s+t) \\
\operatorname{rank}\left(S_{\overline{\mathbf{Y}}_{d}}\right) & =\min (d, s+t) \\
\operatorname{rank}\left(S_{\overline{\mathbf{Y}}_{r}, \overline{\mathbf{Y}}_{d}, \mathbf{X}}\right) & =s+\min (r+d, t) \\
\operatorname{rank}\left(S_{\overline{\mathbf{Y}}_{d}, \mathbf{X}}\right) & =s+\min (d, t)
\end{aligned}
$$

Now, we calculate the rank of $S_{\overline{\mathbf{Y}}_{r} \mid \overline{\mathbf{Y}}_{d}, \mathbf{X}}$. Note that if $d<t$, then $S_{\overline{\mathbf{Y}}_{d}, \mathbf{X}}$ is full rank. If $d \geq t$, we have:

$$
\overline{\mathbf{Y}}_{r}=H_{s r} \mathbf{X}+H_{t r}\left(H_{t d}^{\dagger} H_{t d}\right)^{-1} H_{t d}^{\dagger}\left(\overline{\mathbf{Y}}_{d}-H_{s d} \mathbf{X}\right) \triangleq A\left[\begin{array}{c}
\overline{\mathbf{Y}}_{d} \\
\mathbf{X}
\end{array}\right] .
$$

Therefore, $S_{\overline{\mathbf{Y}}_{r}, \overline{\mathbf{Y}}_{d}, \mathbf{X}}^{(1,2)}=A S_{\overline{\mathbf{Y}}_{d}, \mathbf{X}}$. In either case, the null space of $S_{\overline{\mathbf{Y}}_{d}, \mathbf{X}}$ is a subset of the null space of $S_{\overline{\mathbf{Y}}_{r}, \overline{\mathbf{Y}}_{d}, \mathbf{X}}^{(1,2)}$. Therefore, we have:

$$
\operatorname{rank}\left(S_{\overline{\mathbf{Y}}_{r} \mid \overline{\mathbf{Y}}_{d}, \mathbf{X}}\right)=\operatorname{rank}\left(S_{\overline{\mathbf{Y}}_{r}, \overline{\mathbf{Y}}_{d}, \mathbf{X}}\right)-\operatorname{rank}\left(S_{\overline{\mathbf{Y}}_{d}, \mathbf{X}}\right)
$$

Further, we calculate the rank of $S_{\overline{\mathbf{Y}}_{r} \mid \overline{\mathbf{Y}}_{d}}$. Note that if $d<$ $s+t$, then $S_{\overline{\mathbf{Y}}_{d}}$ is full rank. If $d \geq s+t$, we have:

$$
\overline{\mathbf{Y}}_{r}=\left[\begin{array}{ll}
H_{s r} & H_{t r}
\end{array}\right]\left(H_{d}^{\dagger} H_{d}\right)^{-1} H_{d}^{\dagger} \overline{\mathbf{Y}}_{d} \triangleq B \overline{\mathbf{Y}}_{d}
$$

where $H_{d} \triangleq\left[H_{s d} H_{t d}\right]$. Therefore, $S_{\overline{\mathbf{Y}}_{r}, \overline{\mathbf{Y}}_{d}}^{(1,2)}=B S_{\overline{\mathbf{Y}}_{d}}$. In either case, the null space of $S_{\overline{\mathbf{Y}}_{d}}$ is a subset of the null space of $S_{\overline{\mathbf{Y}}_{r}, \overline{\mathbf{Y}}_{d}}^{(1,2)}$. Therefore, we have:

$$
\operatorname{rank}\left(S_{\overline{\mathbf{Y}}_{r} \mid \overline{\mathbf{Y}}_{d}}\right)=\operatorname{rank}\left(S_{\overline{\mathbf{Y}}_{r}, \overline{\mathbf{Y}}_{d}}\right)-\operatorname{rank}\left(S_{\overline{\mathbf{Y}}_{d}}\right)
$$

To prove the lemma, we consider three different cases: 1) $d<t$, 2) $t \leq d<s+t$, and 3) $d \geq s+t$.

Case 1) When $d<t$, using (21), (22), and (24):

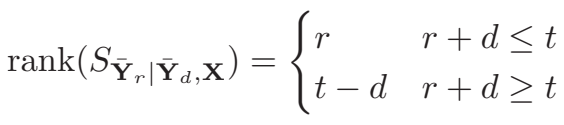

Similarly, using (19), (20), and (26):

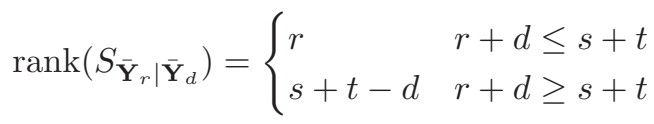

When $r+d \leq t$, both $S_{\overline{\mathbf{Y}}_{r} \mid \overline{\mathbf{Y}}_{d}}$ and $S_{\overline{\mathbf{Y}}_{r} \mid \overline{\mathbf{Y}}_{d}, \mathbf{X}}$ are full rank. Therefore, by the upper bound in (16) we have:

$$
\lim _{\sigma^{2} \rightarrow 0} \lambda_{1} \leq \lim _{\sigma^{2} \rightarrow 0} \frac{\lambda_{1}\left(S_{\mathbf{Y}_{r} \mid \mathbf{Y}_{d}}\right)}{\lambda_{r}\left(S_{\mathbf{Y}_{r} \mid \mathbf{Y}_{d}, \mathbf{X}}\right)}<\infty,
$$

When $r+d>t$, the number of zero eigenvalues of $S_{\overline{\mathbf{Y}}_{r} \mid \overline{\mathbf{Y}}_{d}, \mathbf{X}}$ is more than that of $S_{\overline{\mathbf{Y}}_{r} \mid \overline{\mathbf{Y}}_{d}}$. Therefore, at least for one $i \in$ $\{1, \ldots, r\}$, the limit of the lower bound in (16)

$$
\lim _{\sigma^{2} \rightarrow 0} \frac{\lambda_{i}\left(S_{\mathbf{Y}_{r} \mid \mathbf{Y}_{d}}\right)}{\lambda_{i}\left(S_{\mathbf{Y}_{r} \mid \mathbf{Y}_{d}, \mathbf{X}}\right)}
$$

is equal to infinity. Therefore, $\lim _{\sigma^{2} \rightarrow 0} \lambda_{1}=+\infty$. To summarize, when $d<t$, we have $\lim _{\sigma^{2} \rightarrow 0} \lambda_{1}=+\infty$ if and only if $r+d>t$.

Case 2) When $t \leq d<s+t$, using (21), (22), and (24) we have $\operatorname{rank}\left(S_{\overline{\mathbf{Y}}_{r} \mid \overline{\mathbf{Y}}_{d}, \mathbf{X}}\right)=0$, and using (19), (20), and (26) we have $\operatorname{rank}\left(S_{\overline{\mathbf{Y}}_{r} \mid \overline{\mathbf{Y}}_{d}}\right)>0$. Therefore, similar to (28), we have $\lim _{\sigma^{2} \rightarrow 0} \lambda_{1}=+\infty$.

Case 3) When $d \geq s+t$, using (21), (22), and (24) we have $\operatorname{rank}\left(S_{\overline{\mathbf{Y}}_{r} \mid \overline{\mathbf{Y}}_{d}, \mathbf{X}}\right)=0$, and using (19), (20), and (26) we have

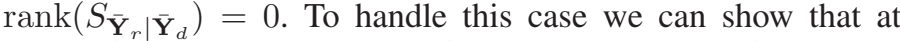
small $\sigma^{2}$, we have $S_{\mathbf{Y}_{r} \mid \mathbf{Y}_{d}} \approx \sigma^{2} S$ and $S_{\mathbf{Y}_{r} \mid \mathbf{Y}_{d} \mathbf{X}} \approx \sigma^{2} S^{\prime}$, where 
$S$ and $S^{\prime}$ are positive definite matrices. Therefore, for some $m, n \in\{1, \ldots, r\}$ we have:

$$
\begin{aligned}
\lim _{\sigma^{2} \rightarrow 0} \lambda_{1}\left(S_{\mathbf{Y}_{r} \mid \mathbf{Y}_{d}} S_{\mathbf{Y}_{r} \mid \mathbf{Y}_{d}, \mathbf{X}}^{-1}\right) & \leq \lim _{\sigma^{2} \rightarrow 0} \frac{\lambda_{1}\left(S_{\mathbf{Y}_{r} \mid \mathbf{Y}_{d}}\right)}{\lambda_{r}\left(S_{\left.\mathbf{Y}_{r} \mid \mathbf{Y}_{d}, \mathbf{X}\right)}\right.} \\
& =\frac{\sigma^{2} \lambda_{m}(S)}{\sigma^{2} \lambda_{n}\left(S^{\prime}\right)}<\infty
\end{aligned}
$$

\section{Connection with Deterministic Relay Channel}

In [5] it has been shown that for a relay channel with a digital link between relay and destination, whenever the observation of the relay is a deterministic function of the observation of destination and the input to the channel, $\mathrm{CF}$ is able to achieve the cut-set bound in (6). In this case, observe that as long as $I\left(\mathbf{X} ; \mathbf{Y}_{r}, \mathbf{Y}_{d}\right)>I\left(\mathbf{X} ; \mathbf{Y}_{d}\right)$ we have $\left.\frac{d \mathcal{C}\left(C_{0}\right)}{d C_{0}}\right|_{C_{0}=0}=1$. However, when $I\left(\mathbf{X} ; \mathbf{Y}_{r}, \mathbf{Y}_{d}\right)=I\left(\mathbf{X} ; \mathbf{Y}_{d}\right)$, e.g., when $\mathbf{Y}_{r}$ is a function of $\mathbf{Y}_{d}$, the relay channel is reversely degraded and the direct transmission achieves the capacity [6]. In this case the slope of interest is 0 .

One can show that, in the high SNR and INR limit, the Gaussian MIMO relay channel considered in this paper has a deterministic component which is not reversely degraded if and only if $r+d>t$ and $d<s+t$. By investing the available capacity $C_{0}$ for quantization of that component, one can achieve the slope of 1 at $C_{0}=0$. This explains the main result of this paper, i.e., $\left.\lim _{\sigma^{2} \rightarrow 0} \frac{d R_{C F}\left(C_{0}\right)}{d C_{0}}\right|_{C_{0}=0}=1$ if and only if $r+d>t$ and $d<s+t$.

To see this more crearly, observe that $\overline{\mathbf{Y}}_{r}$ is a function of $\overline{\mathbf{Y}}_{d}$ and $\mathbf{X}$ if and only if $d \geq t$ in (23); also $\overline{\mathbf{Y}}_{r}$ is a function of $\overline{\mathbf{Y}}_{d}$ if and only if $d \geq t+s$ in (25). Therefore, when $t \leq d<t+s$, the channel defined in (17)-(18) is deterministic, but not reversely degraded. In this case we have $S_{\overline{\mathbf{Y}}_{r} \mid \overline{\mathbf{Y}}_{d} \mathbf{X}}=\mathbf{0}$ and $S_{\overline{\mathbf{Y}}_{r} \mid \overline{\mathbf{Y}}_{d}} \succeq \mathbf{0}$. Thus, $\left.\lim _{\sigma^{2} \rightarrow 0} \frac{d R_{C F}\left(C_{0}\right)}{d C_{0}}\right|_{C_{0}=0}=1$.

Outside of the range $t \leq d<t+s$, it turns out that $\left.\lim _{\sigma^{2} \rightarrow 0} \frac{d R_{C F}\left(C_{0}\right)}{d C_{0}}\right|_{C_{0}=0}=1$ can still be achieved if the channel defined in (17)-(18) has a deterministic component that is not reversely degraded. This happens when $d<t$ and $r+d>t$. Note that under these conditions $S_{\overline{\mathbf{Y}}_{r} \mid \overline{\mathbf{Y}}_{d} \mathbf{X}}$ has singularities and its rank is always smaller than the rank of $S_{\overline{\mathbf{Y}}_{r} \mid \overline{\mathbf{Y}}_{d}}$. The deterministic component is revealed by simultaneous diagonalization of $S_{\mathbf{Y}_{r} \mid \mathbf{Y}_{d}, \mathbf{X}}$ and $S_{\mathbf{Y}_{r} \mid \mathbf{Y}_{d}}$.

If the conditions $r+d>t$ and $d<t+s$ are not satisfied, the limit of the slope of interest is strictly less than 1 . To see this, note that when $r \leq t-d$, the interference signal makes both $S_{\overline{\mathbf{Y}}_{r} \mid \overline{\mathbf{Y}}_{d} \mathbf{X}}$ and $S_{\overline{\mathbf{Y}}_{r} \mid \overline{\mathbf{Y}}_{d}}$ full rank; thus, the channel (17)-(18) cannot have a deterministic component. When $d \geq s+t, \overline{\mathbf{Y}}_{r}$ is a function of $\overline{\mathbf{Y}}_{d}$; therefore, the channel (17)-(18) is reversely degraded. In both cases, $\left.\lim _{\sigma^{2} \rightarrow 0} \frac{d R_{C F}\left(C_{0}\right)}{d C_{0}}\right|_{C_{0}=0}<1$.

\section{Numerical EXAMPLE}

In this section we numerically evaluate the slope of $R_{C F}\left(C_{0}\right)$ as a function of $(r, d, s, t)$ in a practical scenario. Consider a picocell deployment with a pico-base-station with $s=4$ antennas transmitting 1 Watt of power over $10 \mathrm{MHz}$ to a user

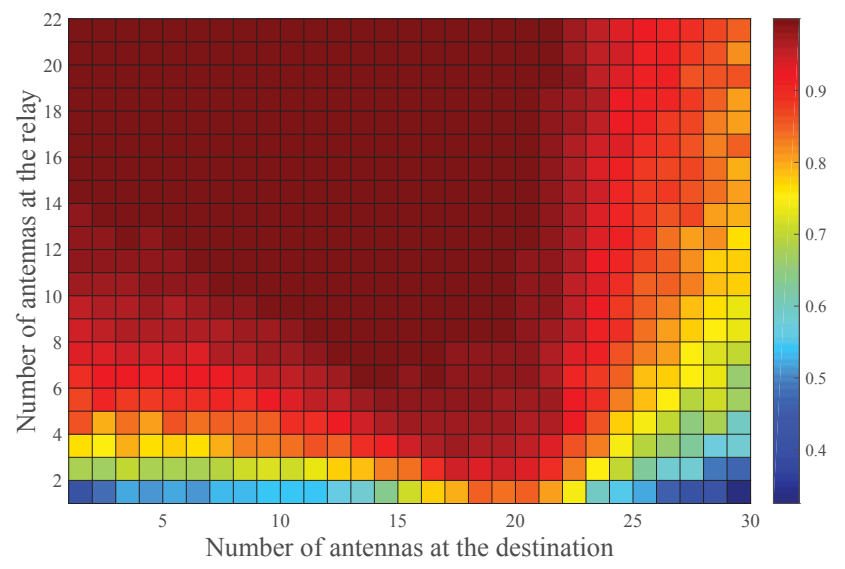

Fig. 2. The average value of $R_{C F}(1 \mathrm{Mbps})-R_{C F}(0)$ in Mbps as number of antennas at the destination and relay varies with $s=4$ antennas at source and 18 single-antenna interference sources $t=18$

distance $100 \mathrm{~m}$ away. We include 18 single-antenna pico-basestations $200 \mathrm{~m}$ away from each other on a hexagonal grid at the same power level. A second user located nearby acts as a relay as shown in Fig. 1. The background noise power spectral density is assumed to be $-170 \mathrm{dBm} / \mathrm{Hz}$. A channel model with pathloss exponent of 3.76 and $8 \mathrm{~dB}$ shadowing is used. Fig. 2 illustrates the average improvement in achievable rate by $\mathrm{CF}$ with $C_{0}=1 \mathrm{Mbps}$ as a function of $r$ and $d$. Fig. 2 shows that this slope is close to 1 when approximately $r+d>18$ and $d<22$, as predicted by Theorem 1 .

\section{CONCLUSION}

This paper characterizes the slope of $\mathrm{CF}$ rate versus $C_{0}$ at $C_{0}=0$ for the Gaussian MIMO relay channel with correlated noises. We show that this slope asymptotically approaches its maximum value of 1 at high SNR and INR, if and only if the number of antennas in the network is such that full cooperation between relay and destination improves the maximum spatial DoF of the channel.

\section{REFERENCES}

[1] S. Simoens, O. Muoz-Medina, J. Vidal, and A. del Coso, "Compress-andforward cooperative MIMO relaying with full channel state information," IEEE Trans. Signal Process., vol. 58, no. 2, pp. 781-791, Feb. 2010.

[2] A. del Coso and S. Simoens, "Distributed compression for MIMO coordinated networks with a backhaul constraint," IEEE Trans. Wireless Commun., vol. 8, no. 9, pp. 4698-4709, Sep. 2009.

[3] S. A. Ayoughi and W. Yu, "Optimized MIMO transmission and compression for interference mitigation with user cooperation," in Proc. IEEE Int. Conf. Commun. (ICC) 2015.

[4] C. Tian and J. Chen, "Remote vector Gaussian source coding with decoder side information under mutual information and distortion constraints," IEEE Trans. Inf. Theory, vol. 55, no. 10, pp. 4676-4680, Oct. 2009.

[5] T. M. Cover and Y. Kim, "Capacity of a class of deterministic relay channel," in Proc. Int. Symp. Inf. Theory (ISIT), Jun. 2007, pp. 591-595.

[6] T. Cover and A. El Gamal, "Capacity theorems for the relay channel," IEEE Trans. Inf. Theory, vol. 25, no. 5, pp. 572-584, Sep. 1979.

[7] L.-Z. Lu and C. E. M. Pearce, "Some new bounds for singular values and eigenvalues of matrix products," Ann. Operations Res., vol. 98, no. 1-4, pp. 141-148, Dec. 2000.

[8] D. Carlson, E. Haynsworth, and T. Markham, "A generalization of the Schur complement by means of the Moore-Penrose inverse," SIAM J. Appl. Math., vol. 26, no. 1, pp. 169-175, Jan. 1974. 